became better known. Disease of the nervous system has been reported in both the venereally acquired and the dysenteric varieties of the disease, and usually takes the form of peripheral neuritis. Pericarditis and myocarditis with conductive defects accur in about $7 \%$ of cases in the acute stage, and aortic incompetence may develop later. ${ }^{2-5}$ Pleurisy may occur but is uncommon. The fact that some patients with Reiter's disease develop ankylosing spondylitis and that most patients with typical ankylosing spondylitis have evidence of genital infection $^{6-8}$ has led to speculation on the relationship between the two, but the matter remains unsettled. Recently S. S. Bleehen and colleagues ${ }^{9}$ described the case of a patient who died from amyloid disease of intestines, liver, spleen, adrenals, and kidneys two and a half years after the onset of a severe attack of Reiter's disease ; while in a series of 302 consecutive patients with Reiter's disease G. Csonka ${ }^{10}$ found that ten patients developed thrombophlebitis of the deep veins of the legs a few days after the onset of arthritis.

Of the many unanswered questions relating to this disease the most important is that of the cause. Reiter's disease itself has been regarded as a complication of the common condition called non-gonococcal, non-specific, or abacterial urethritis. Search for the cause of this condition has, for the most part, centred on the mycoplasma group of organisms and on viruses. But despite much work on the subject ${ }^{11-18}$ the part played by the various organisms isolated from patients remains an open question. The strong evidence in favour of venereal transmission suggests that it is an infection, and the clinical course of the disease, the cellular content of the discharge, and the response to skin tests with various microorganisms give no confirmation of the suggestion that it is an allergic process. Moreover, treatment with antihistamines and corticosteroids neither cures nor relieves the genital condition. Further advances in diagnosis and treatment await discovery of the cause, and the answer to this problem should increase our understanding of the causation of rheumatic dtseases in general.

\section{Oral Contraceptives and Tests of Thyroid Function}

There are many snags for the unwary in interpreting tests of thyroid function. It is important, therefore, that the dinician ordering such tests should be aware of factors which can give results at variance with the true thyroid status of the patient.

The most common interfering factor is exposure of the patient to iodine-containing drugs or contrast media. These block thyroid uptake of radioiodine and may raise the concentration of plasma protein-bound iodine (P.B.I.). Under normal conditions the P.B.I. is a reliable quantitative index of thyroid function, representing mainly the circulating thyroid hormone. In the euthyroid state it ranges between 3 and $7.5 \mu \mathrm{g}$. per $100 \mathrm{ml}$. plasma, varying slightly between different laboratories and methods of estimation. Figures above this level are consistent with thyrotoxicosis and below with hypothyroidism. The borderline between the normal and thyrotoxic state is less clearly defined than that between normal and hypothyroid. ${ }^{1}$
Other factors besides exogenous iodide tend to raise the P.B.I. in healthy persons and so render the test invalid as a true measure of thyroid function. It has long been known that increased levels of oestrogen in the blood, whether exogenous or, as in pregnancy, endogenous, will raise the P.B.I. to levels typical of thyrotoxicosis. This happens without any increase in uptake of radioiodine by the thyroid gland and, except in pregnancy, without a rise in the basal metabolic rate. Oestrogens do this by increasing the binding capacity of thyroxine-binding globulin for thyroid hormone. ${ }^{2}{ }^{3}$ This capacity is less for triiodothyronine than for thyroxine. The resultant increase in bound (inactive) hormone occurs without increase in concentration of free hormone; and, because thyroxine and triiodothyronine enter the cells only in free form, there are no manifestations of thyrotoxicosis. Similarly oestrogens interfere with tests of thyroid function based on uptake of radioactive triiodothyronine by the patient's red cells or by resin incubated in the patient's serum. The uptake is diminished owing to increase in the proportion of bound to unbound triiodothyronine, giving results consistent with hypothyroidism.

Among oestrogens that may affect these tests are the oral contraceptives. All of these are compounded of a progestogen and an oestrogen, each of which may vary in structure and quantity as between different preparations now in use. Progestogens seem to have little if any effect on P.B.I. or other tests of thyroid function, so it can be assumed that any such action of these pills is due to the oestrogen component. It has been reported that in women taking oral contraceptives containing $0.05 \mathrm{mg}$. ethinyl oestradiol there is no increase in P.B.I. ${ }^{4}$ However, when the dose of ethinyl oestradiol is 0.075 mg. ${ }^{5}{ }^{6}$ or of ethinodiol diacetate $1 \mathrm{mg} .{ }^{7}$ or of mestranol $0.075-$ $0.1 \mathrm{mg} .{ }^{8}$ all reports have shown an increase in P.B.I. in a large proportion of women.

Recently D. Winikoff and K. Taylor ${ }^{9}$ have studied the short- and long-term effects of a wide variety of oral contraceptives on various indices of thyroid function. They found that each of ten different preparations caused an increase in P.B.I. and a fall in the uptake of radioactive triiodothyronine by resin. Thyroid uptake of radioiodine at four hours was unaltered. The changes were proportional to the quantity of oestrogen in the preparations used. After one cycle of administration the changes continued for three to seven days, with return to normal four weeks after cessation of treatment. During long-term administration the effects were slightly greater, and return to normal took at least six weeks after the woman ceased taking the contraceptive pills. The magnitude of these changes, though not nearly so great as in pregnancy, were sufficient to bring many of the test results into the thyrotoxic range.

Thus in women of child-bearing age undergoing tests of thyroid function it is essential to know whether they are taking oral contraceptives. When they are, radioiodine uptake by the thyroid gland is the most reliable test.

Radcliff, F. J., Baker, J. M., Croydon, M. J., Hart, M. J., and Hales, I. B., 7. clin. Endocr., 1964, 24, 883.

2 Dowling, J. T., Freinkel, N., and Ingbar, S. H., ibid., 1956, 16, 1491 .

3 Tata, J. R., Brit. med. Bull., 1960, 16, 142.

Walser, H. C., Margulis, R. R., and Ladd, J. E., Int. F. Fertil., 1964, 9, 189.

5 Hollander, C. S., Garcia, A. M., Sturgis, S. H., and Selenkow, H. A., New Engl. F. Med., 1963, 269, 501.

- Larsson-Cohn U., Lancet, 1965, 1, 317.

' Pincus, G., Searle Symposium, 1964. Melbourne.

${ }^{8}$ Florsheim, W. H., and Faircloth, M. A., Proc. Soc. exp. Biol. (N.Y.), $1964,117,56$.

'Winikoff, D., and Taylor, K., Med. F. Aust., 1966, 2, 108. 